\title{
Evaluation of Implanted Stent Occlusion Status Based on Neointimal Tissue Bioimpedance Simulations
}

\author{
J. M. Portillo-Anaya, ${ }^{1}$ P. Pérez, ${ }^{1,2}$ A. Olmo $\mathbb{D}^{1,2}$ G. Huertas, ${ }^{1,3}$ and A. Yúfera $\mathbb{D}^{1,2}$ \\ ${ }^{1}$ Instituto de Microelectrónica de Sevilla, Universidad de Sevilla (CSIC-US), Av. Américo Vespucio, 28, Sevilla 41092, Spain \\ ${ }^{2}$ Departamento de Tecnología Electrónica, Escuela Técnica Superior de Ingeniería Informática, Universidad de Sevilla, \\ Av. Reina Mercedes, SN, Sevilla 41012, Spain \\ ${ }^{3}$ Departamento de Electrónica y Electromagnetismo, Facultad de Física, Universidad de Sevilla, Av. Reina Mercedes, SN, \\ Sevilla 41012, Spain \\ Correspondence should be addressed to A. Yúfera; yufera@imse-cnm.csic.es
}

Received 27 March 2019; Revised 15 October 2019; Accepted 6 November 2019; Published 5 December 2019

Academic Editor: Roberto Paolesse

Copyright (C) 2019 J. M. Portillo-Anaya et al. This is an open access article distributed under the Creative Commons Attribution License, which permits unrestricted use, distribution, and reproduction in any medium, provided the original work is properly cited.

\begin{abstract}
This paper describes the characterization of the light hole, also known as the lumen, in implanted stents affected by restenosis processes using bioimpedance (BI) as a biomarker. The presented approach will enable real-time monitoring of lumens in implanted stents. The basis of the work hereby reported is the fact that neointimal tissues involved in restenosis can be detected and measured through their impedance properties, namely, conductivity and permittivity. To exploit these properties, a 4electrode setup for BI measurement is proposed. This setup allows study of the influence of the various tissues involved in restenosis fat, muscle, fibre, and endothelium, together with the blood, on the BI value at several frequencies. In addition, BI simulation tests were performed using the electric physics module available in COMSOL Multiphysics ${ }^{\circledR}$. Interestingly, fat constitutes the most influential layer on the value of impedance (measured in $\mathrm{k} \Omega / \mu \mathrm{m}$-magnitude change per micrometre of lumen occlusion). A case study using a standard stent is also presented. In this study, where the involved tissues and blood were simultaneously considered, we conducted an analysis for stable and vulnerable plaques in restenosis test situations. In this regard, the proposed method is useful to test the stent obstruction and detect potential dangerous cases due to nonstable fat accumulation.
\end{abstract}

\section{Introduction}

Ischemic heart disease is the main cause of morbidity and mortality in the world $[1,2]$. The interior of the elastic and muscular arteries shrinks (stenosis), mostly because of the growth of a lipid layer caused by an illness called atherosclerosis. This gives rise to angina chest pain and acute myocardial infarction. A $50 \%$ occlusion is already considered worrisome [3]. There are certain regions prone to exceeding that threshold, such as the Left Anterior Descending (LAD) coronary artery [3]. Among the existing treatments, the most popular one employs a flexible metal mesh (stent) to mechanically support the opening of stenotic segments $[4$, 5]. However, despite improvements on surgery techniques and stent design [6], there is a tendency for medium-term restenosis to eventually occur [7], as shown in Figure 1, which displays the cross-sectional view of an implanted stent covered by a neointima layer. For this reason, it is necessary to carry out a follow-up plan, which costs between $\$ 10,000$ and $\$ 17,000$ per patient for only the first year [8] — provided that patients do not require more revascularization procedures. Remarkably, only in the United States, approximately 600,000 patients are stented every year [8]. In addition to the high expenses, the techniques applied to follow-up restenosis are either invasive or ionising [3].

Real-time characterization of neointima tissue through a smart stent would ease the integration of readout circuits to measure and compensate the sensitivity loss of pressure sensors suggested in previous works [9-11]. In this regard, different studies have recently proposed BI techniques to 


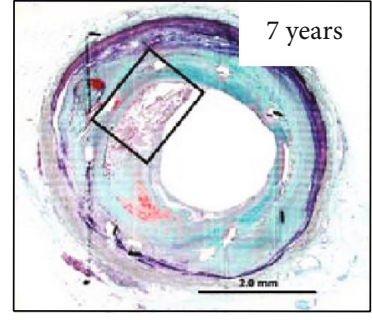

(a)

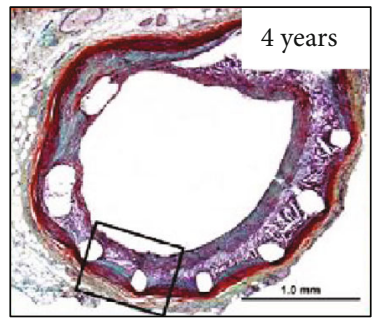

(c)

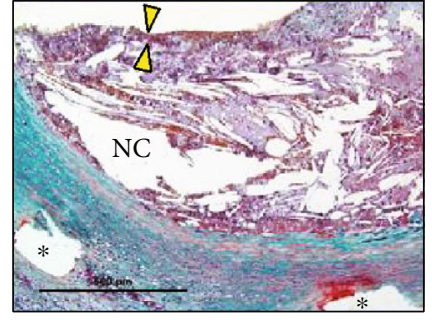

(b)

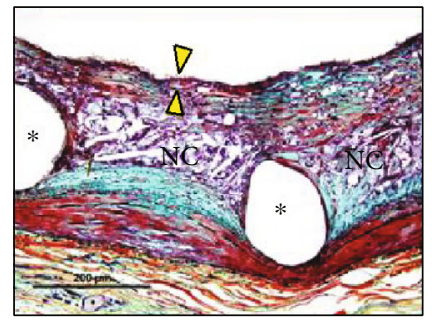

(d)

Figure 1: Restenosis in an implanted stent after ( $a, b) 7$ years and (c, d) 4 years, from [7]. Note that (b) and (d) are zoomed images from (a) and (c), respectively. The asterisks inside the holes in (b) and (d) represent the stent metal struts. The yellow arrows indicate the thinnest part of a fibre layer. NC: necrotic cores.

follow-up restenosis by analysing the growth of the neointima layer. Some of these techniques consider the use of medical invasive instruments such as a catheter with a matrix of microelectrodes placed near the tip to measure the damaged area [12]. Another approach estimates the influence of fat tissue with electrical simulations oriented to measure the BI inside a stent [13]. The use of the stent itself as a large electrode has also been proposed [14]. In addition to monitoring the restenosis in coronary arteries, the aforementioned technologies can be useful for other body parts or illnesses such as the femoropopliteal disease [15] or even for other purposes, e.g., neural recording [16]. The results obtained in these previous works that explored BI techniques were notable and promising. However, they still need catheterization to connect to external measurement equipment. This is a fundamental difference with respect to the work presented in this paper.

There exist methods to monitor cell cultures in vivo based on their BI [17-20]. In [17], a cell-culture was transformed into a "biological oscillator" whose characteristic parameters (i.e., frequency, amplitude, phase, etc.) are related with the cell-culture evolution and can be easily determined. This approach, known as oscillation-based test (OBT), can also be valid to monitor the growth of neointimal tissue. A feedback-loop closed-system topology injects a quasisinusoidal signal to stimulate the tissue. An input resistor keeps the current injected to the blood vessel within safety margins. Relevant parameters are extracted from the frequency and amplitude of the oscillations. In [20], primary cells were also cultured and characterized while conforming to the endothelium tissue.

Overall, this study is primarily focused on the development of a setup for neointimal tissue characterization using finite element analysis (FEA) simulations to measure its BI. We also address the application of this setup to the tissues composing a neointima layer to check their influence on the restenosis process. The FEA includes a case study with a stent to characterize its lumen with stable and vulnerable plaques in restenosis situations. The paper is organized as follows. Materials and Methods describes how the finite element simulations that consider the electrical properties of different tissues were performed with COMSOL Multiphysics ${ }^{\circledR}$. We also describe the electrode setup selected for optimum BI test. Results and Discussion presents simulations for various cases of the involved tissues and reports the performance obtained for a particular stent case. Finally, Conclusions highlights the most relevant points of our work.

\section{Materials and Methods}

2.1. Simulation Tool. COMSOL Multiphysics ${ }^{\circledR}$ is a standard tool for FEA and simulation of structures, useful to evaluate physical phenomena about them. In our case, for a particular tissue under test, an AC electric current at a given frequency is applied between two input electrodes, and the voltage response is measured by two output electrodes, which are considered as high-impedance nodes [21, 22]. The voltageto-current ratio (i.e., the $\mathrm{BI}$ ) is measured at the output electrodes for each frequency, obtaining its magnitude and phase. (Real and imaginary impedance components can also be obtained, but this study focuses on magnitude and phase as the parameters for describing the BI.) This electrical test is reproduced through FEA by defining the full structure, i.e., electrodes plus tissues and blood, and applying the physical conditions derived from the test itself.

2.2. Tissue and Blood Model. Neointimal tissue grows over the interior of stents. It is structured in several stacked layers of tissues, namely, the fat, muscle, fibre, and endothelium, filled by the blood. These layers appear progressively over 
time, defining a restenosis process. Each one has its own electrical properties, derived from its electrical parameters, that is, conductivity $(\sigma)$ and permittivity $(\varepsilon)$ [23], which depend on frequency. The geometry of each layer as well as the assignment of the corresponding conductivity and permittivity at each operation frequency must be incorporated into the FEA $[18,19,24]$. Thus, the analysis is carried out for several types and sizes of tissues, reproducing the restenosis phenomenon for a given setup test. Tissue thickness can range over several tens of micrometres $(\mu \mathrm{m})$, except for the endothelium, which only comprises a $10 \mu \mathrm{m}$ thick cell monolayer. The corresponding electrical parameters, extracted from [23], are listed in Tables 1 and 2 for frequencies ranging from $1 \mathrm{~Hz}$ to $1 \mathrm{MHz}$.

2.3. Electrode Setup. BI is commonly measured through either a 2-electrode or a 4-electrode setup. The main advantage of the 4-electrode setup is that the voltage response is not dependent on the electrode impedance, provided that it is measured by a high-input-impedance amplifier. In this case, a 4-wire system is selected for measuring the tissue or biological layers such that 2 wires are used for input current excitation and 2 wires are employed for measurement of the output voltage response. Several configurations have been analysed for this 4-wire setup, considering that the electrodes can be aligned or not. As a result of such analyses, the configuration shown in Figure 2 was selected, as it reduces the leakage associated to the field lines that are not sensed by the output electrodes. A better impedance test is thus achieved because the electrodes for measurement of the output voltage sense parallel field current lines. The setup dimensions, described in Figure 2, were established according to the average LAD diameter [24].

The dimensions of the gold electrodes were set to $7 \mu \mathrm{m}$ $\times 7 \mu \mathrm{m} \times 2.5 \mu \mathrm{m}$. The electrodes were deposited over an electrically insulated material employed for setup support and insulation. The frequency values set for FEA ranged from $1 \mathrm{~Hz}$ to $1 \mathrm{MHz}$, with a current excitation amplitude of $3 \mu \mathrm{A}$.

\section{Results and Discussion}

As previously mentioned, FEA was performed with the COMSOL Multiphysics ${ }^{\circledR}$ computer program [19]. For each simulation, and therefore, for each frequency, a geometric (size) and electrical (conductivity and permittivity) description of the tissue layer under test was introduced. The AC current amplitude was set to $3 \mu \mathrm{A}$, and the voltage response at the output electrodes was measured to calculate the magnitude and phase of the sample under test. We considered four types of simulations-single tissue, restenosis tissues, symmetrical restenosis, and nonsymmetrical restenosis-for characterization of neointimal tissue according to the selected features of the tissue layer.

3.1. Single Tissue. We simulated changes in layer thickness ( $h$ in Figure 2) for the tissues and operation frequencies listed in Tables 1 and 2. A parametric sweep was performed for every single tissue in each simulation. To this end, the layer thickness was assumed to vary according to a prism made by the
TABLE 1: Electric conductivity, $\sigma(\mathrm{S} / \mathrm{m})$, for tissues involved in the neointimal layer and blood, employed for FEA in the frequency range $\left(1,10^{6}\right) \mathrm{Hz}$.

\begin{tabular}{lccccc}
\hline Frequency $(\mathrm{Hz})$ & Fat & Muscle & Fibre & Endothelium & Blood \\
\hline $10^{0}$ & 0.035 & 0.202 & 0.25 & 0.0500 & 0.700 \\
$10^{1}$ & 0.038 & 0.202 & 0.25 & 0.0500 & 0.700 \\
$10^{2}$ & 0.041 & 0.267 & 0.25 & 0.0500 & 0.700 \\
$10^{3}$ & 0.042 & 0.321 & 0.25 & 0.0500 & 0.700 \\
$10^{4}$ & 0.043 & 0.341 & 0.25 & 0.0500 & 0.700 \\
$10^{5}$ & 0.043 & 0.362 & 0.30 & 0.0502 & 0.702 \\
$10^{6}$ & 0.044 & 0.503 & 0.35 & 0.1460 & 0.840 \\
\hline
\end{tabular}

respective tissues. Thus, the thickness value ranged from 25 to $200 \mu \mathrm{m}$, with a step of $25 \mu \mathrm{m}$, while keeping the prism depth and width constant, $20 \mathrm{~mm}$ and $2 \mathrm{~mm}$, respectively. The electrode setup location is shown in Figure 2. Once data were collected and ordered, the magnitude and phase impedances were depicted to show their respective variations as a function of tissue thickness and frequency.

Figures 3-7 represent the magnitude and phase for the BI measured through FEA at the output electrodes. The magnitude is represented in 3D plots as a function of thickness $(h)$ and frequency. The phase is plotted only as a function of frequency because the influence derived from thickness changes is not substantial. This is the reason why thickness has not been considered for phase plots. In general, large BI magnitude values are observed for small values of $h$. This implies lower cross-section surfaces in the normal direction of current line paths. Besides, the values of BI are larger for lower frequencies. Phase shifts are small, below $12^{\circ}$ for all cases, being more sensitive for muscle (Figure 4(b)) and fat (Figure 3(b)) tissues, in this order.

From these BI spectroscopy curves, we evaluated the sensitivity or influence of each tissue in relation to thickness increments for every frequency. In this way, we can infer which one provides better information or has a greater influence over the restenosis process. This evaluation was focused on the magnitude, which seems to be the most influenced component of the BI by changes in tissue thickness and frequency. The maximum (and minimum) changes in magnitude for the involved tissues are depicted in Figure 8 (expressed in $\mathrm{k} \Omega$ per $\mu \mathrm{m}$ of thickness increment in every frequency decade) for the proposed 4-electrode setup. Note from these curves that the greatest sensitivity is obtained for fat tissue (Figure 8(a)), while the magnitude changes for fibre and endothelium layers are lower and constant in frequency. In general, the sensitivity is greater for low frequencies. Changes associated to the endothelium have a low influence because this $10 \mu \mathrm{m}$ thick cell monolayer never varies. However, its influence is present from the beginning of the restenosis process.

3.2. Restenosis Tissues. Impedance variation as a function of tissue thickness and frequency is presented in Table 3 and Figures 9 and 10, respectively. The difference between these results and those of the previous section is that now all the tissues were simultaneously taken into account. Thus, the 
TABLE 2: Electric relative permittivity, $\varepsilon_{r}$, for tissues involved in the neointimal layer and blood, employed for FEA in the frequency range (1, $\left.10^{6}\right) \mathrm{Hz}$.

\begin{tabular}{lccccc}
\hline Frequency $(\mathrm{Hz})$ & Fat & Muscle & Fibre & Endothelium & Blood \\
\hline $10^{0}$ & $9.91 \times 10^{6}$ & $2.62 \times 10^{7}$ & $2 \times 10^{4}$ & $3.61 \times 10^{3}$ & $5.26 \times 10^{3}$ \\
$10^{1}$ & $5.03 \times 10^{6}$ & $2.57 \times 10^{7}$ & $2 \times 10^{4}$ & $3.61 \times 10^{3}$ & $5.26 \times 10^{3}$ \\
$10^{2}$ & $1.52 \times 10^{5}$ & $9.33 \times 10^{6}$ & $1 \times 10^{4}$ & $3.61 \times 10^{3}$ & $5.26 \times 10^{3}$ \\
$10^{3}$ & $1.93 \times 10^{4}$ & $4.35 \times 10^{5}$ & $1 \times 10^{3}$ & $3.61 \times 10^{3}$ & $5.26 \times 10^{3}$ \\
$10^{4}$ & $9.12 \times 10^{2}$ & $2.59 \times 10^{4}$ & $1 \times 10^{2}$ & $3.61 \times 10^{3}$ & $5.26 \times 10^{3}$ \\
$10^{5}$ & $1.01 \times 10^{2}$ & $8.09 \times 10^{3}$ & $2 \times 10^{1}$ & $3.58 \times 10^{3}$ & $5.22 \times 10^{3}$ \\
$10^{6}$ & $5.08 \times 10^{1}$ & $1.84 \times 10^{3}$ & $1 \times 10^{1}$ & $1.85 \times 10^{3}$ & $3.12 \times 10^{3}$ \\
\hline
\end{tabular}

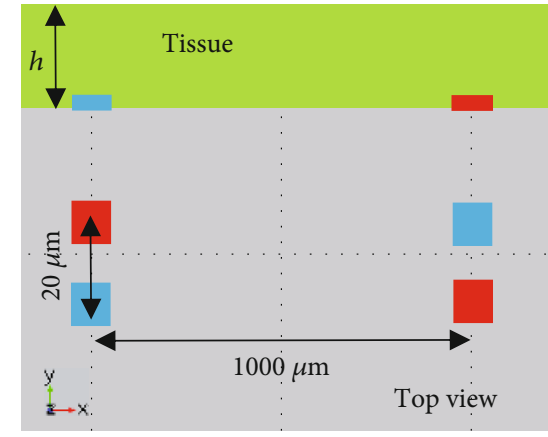

(a)
Cross-section view

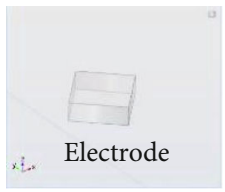

(b)

Figure 2: (a) Top- and cross sections for a 4-electrode setup placed on top of an insulating layer. Two electrodes are used for current excitation (blue) and two electrodes for BI measurement (red); $h$ : tissue thickness. (b) Gold electrode $(7 \mu \mathrm{m} \times 7 \mu \mathrm{m} \times 2.5 \mu \mathrm{m})$.

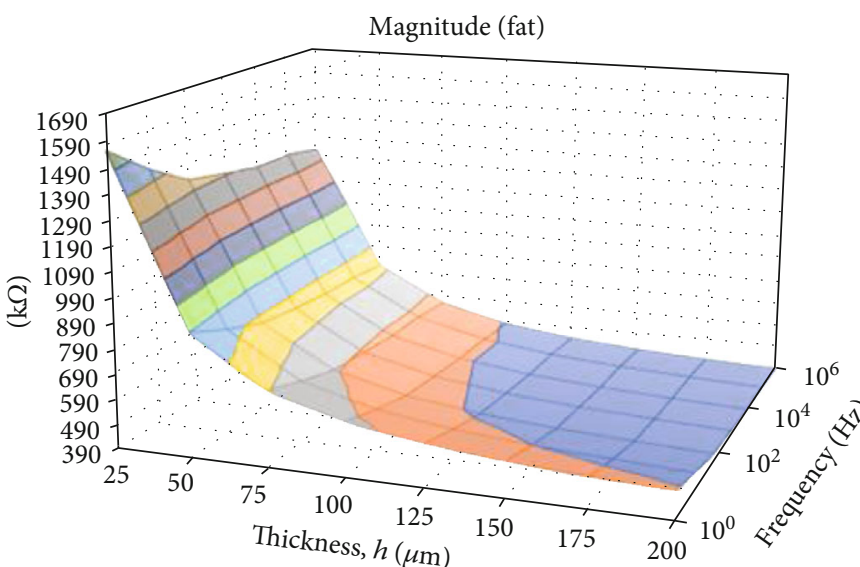

$\mathrm{k} \Omega$

$\square 1590-1690$

$\square$ 1490-1590

$\square$ 1390-1490

$\square 1290-1390$

$\square$ 1190-1290

$\square 1090-1190$

口990-1090

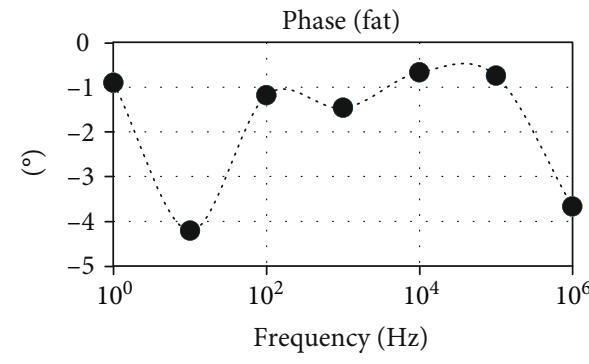

$\square 890-990$

$\square 790-890$

$\square 690-790$

$\square 590-690$

$\square 490-590$

$\square 390-490$

(a)

(b)

FIGURE 3: Magnitude and phase of the impedance in the fat layer. 

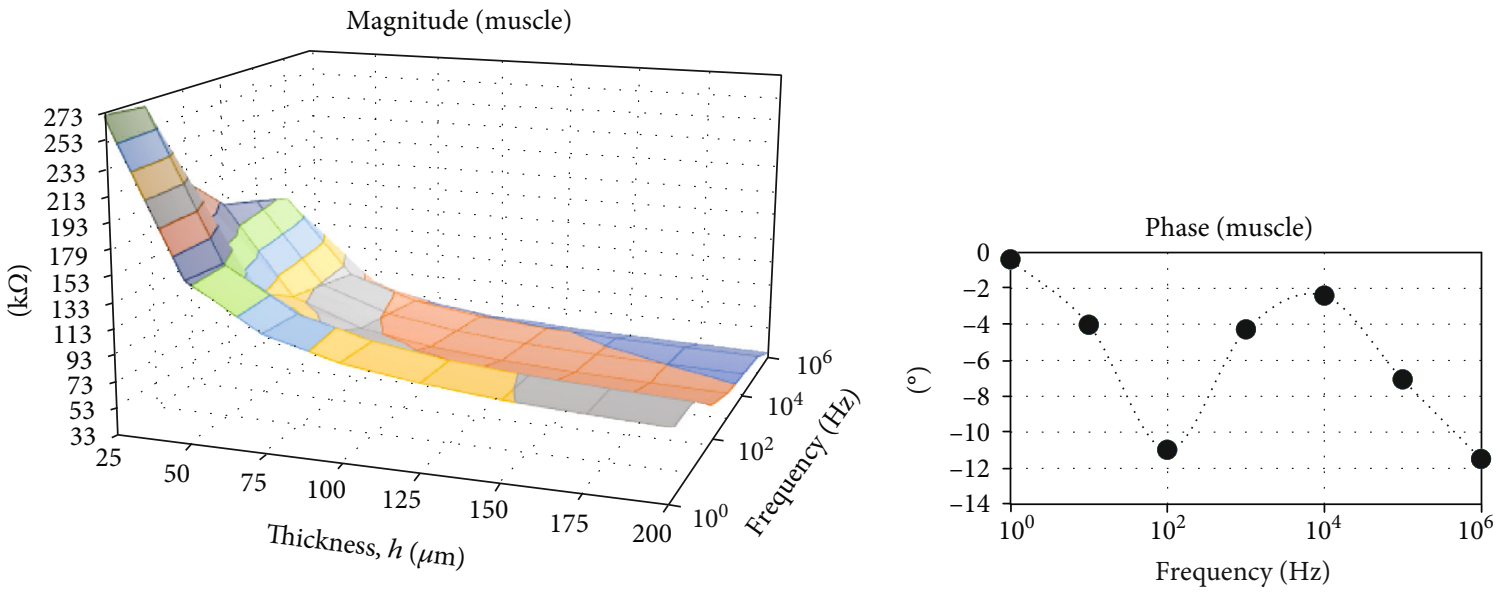

$\mathrm{k} \Omega$

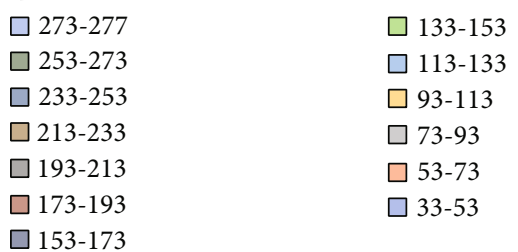

(a)

(b)

Figure 4: Magnitude and phase of the impedance in the muscle layer.

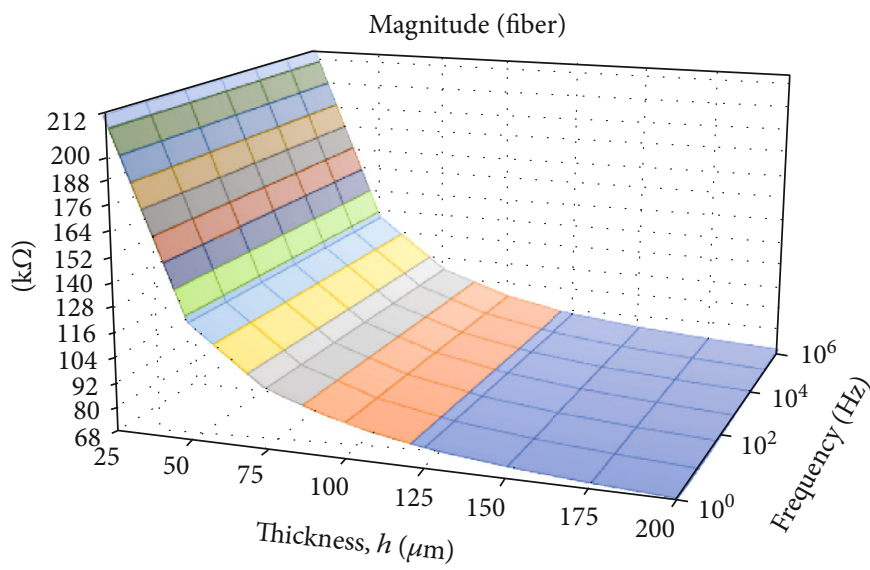

$\mathrm{k} \Omega$

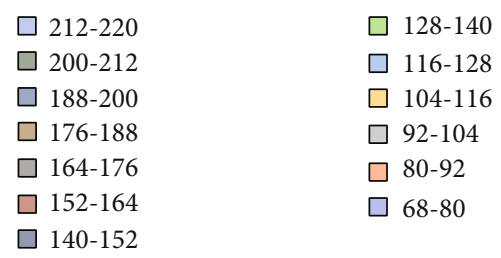

FIGURE 5: Magnitude and phase of the impedance in the fibre layer.

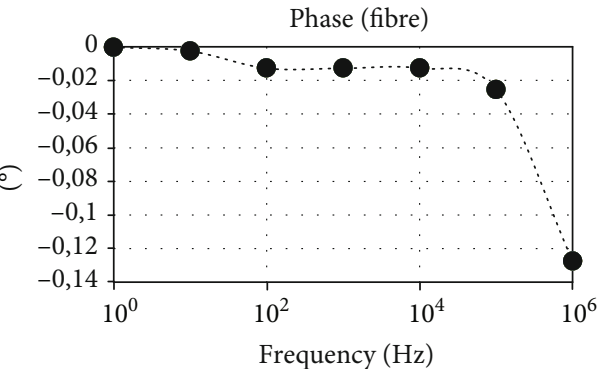

Frequency $(\mathrm{Hz})$ thickness changed differently, as shown in Table 3. Here, the representation of a supposed coronary lumen (the average LAD size was taken as a model) becoming narrower was emulated, choosing a specific stent for this case $[25,26]$ with a vessel radius of $1150 \mu \mathrm{m}$. Cases 1 to 9 in Table 3 represent an example of normal evolution of a restenosis process according to the criteria in [28]. In a primary phase, the neointimal layer comprises the endothelium and smooth muscle. This corresponds to initial cases 1 and 2. Then, the fat layer starts to grow (case 3) around the electrodes, together with the fibre and muscle, which are moved to the medium layer (cases 3-4 to case 9). The muscle and fat 

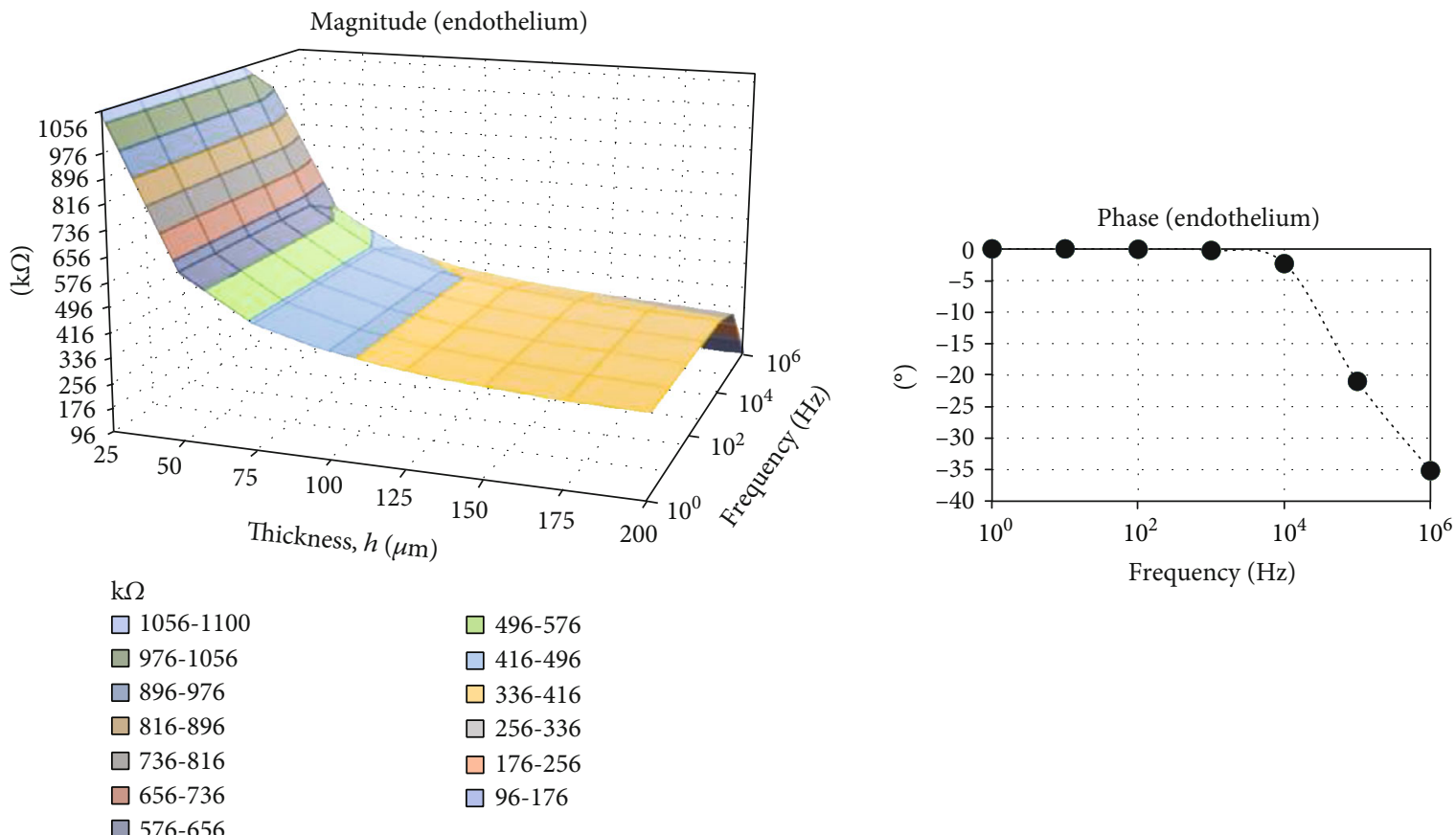

Figure 6: Magnitude and phase of the impedance in the endothelium layer.
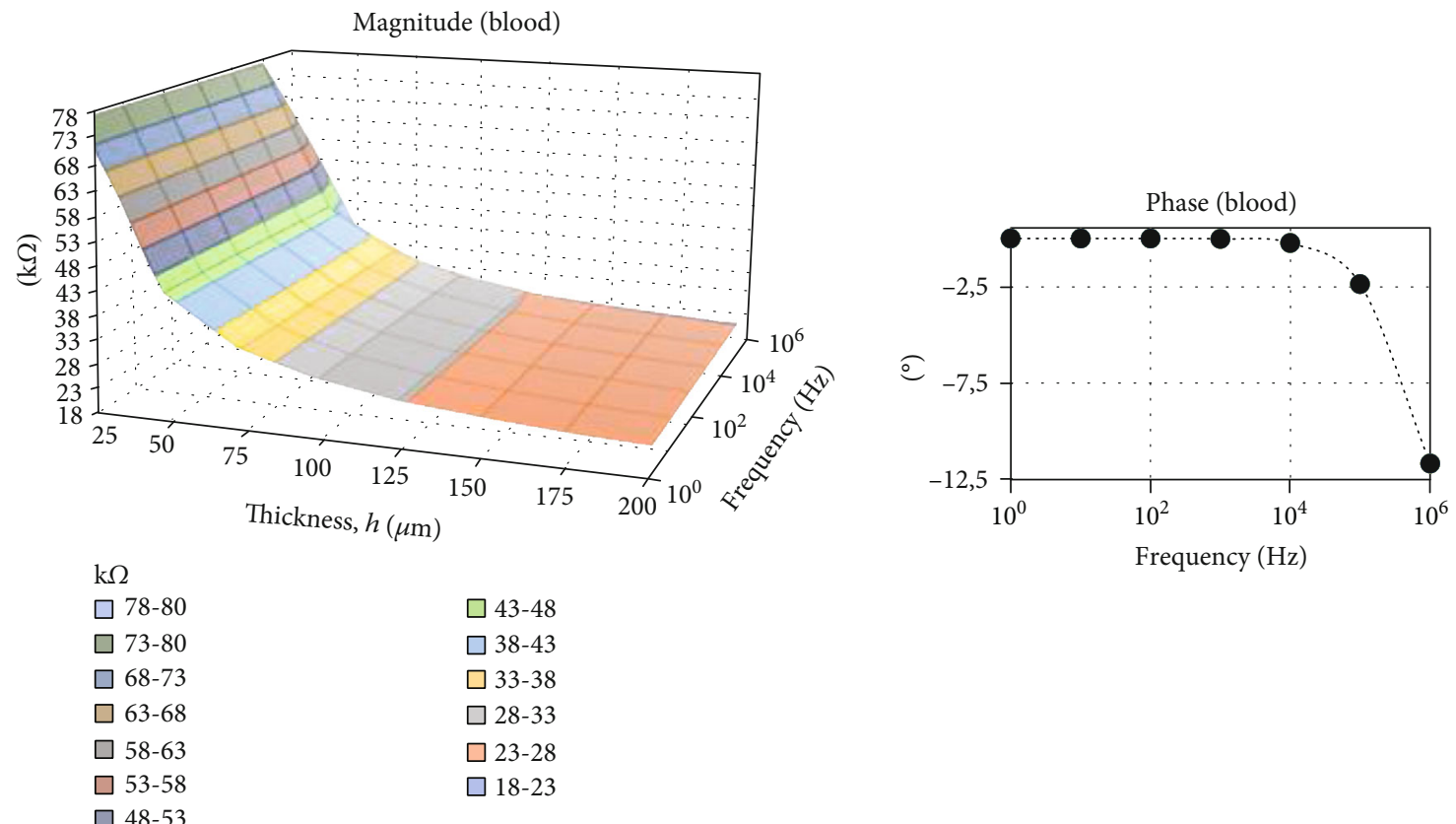

FIGURE 7: Magnitude and phase of the impedance in the blood.

growth ratios are similar and correspond to the data evolution described in [28].

These nine different cases were simulated taking into account the increase in occlusion percentage. Thus, the reported plots (magnitude and phase) illustrate how impedance varied as a function of frequency for increasing values of vessel occlusion (Figures 9(a) and 9(b), respectively).
Figure 9(a) shows that, concerning magnitude changes, there exists a remarkable sensitivity to occlusion increments. In addition, the maximum impedance magnitude sensitivity was evaluated for the cases proposed in Table 3. The results are presented in Figure 10. In particular, Figure 10(a) represents the maximum and minimum changes of the impedance magnitude per micrometre increment for the cases listed in 


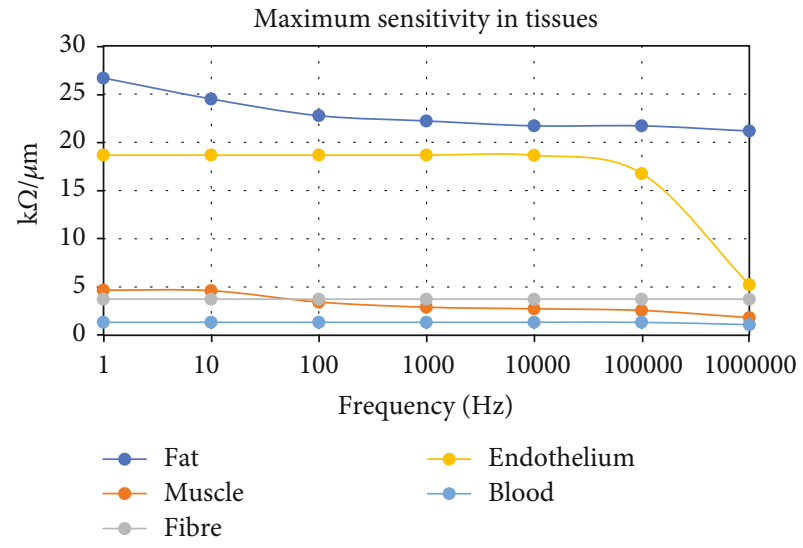

(a)

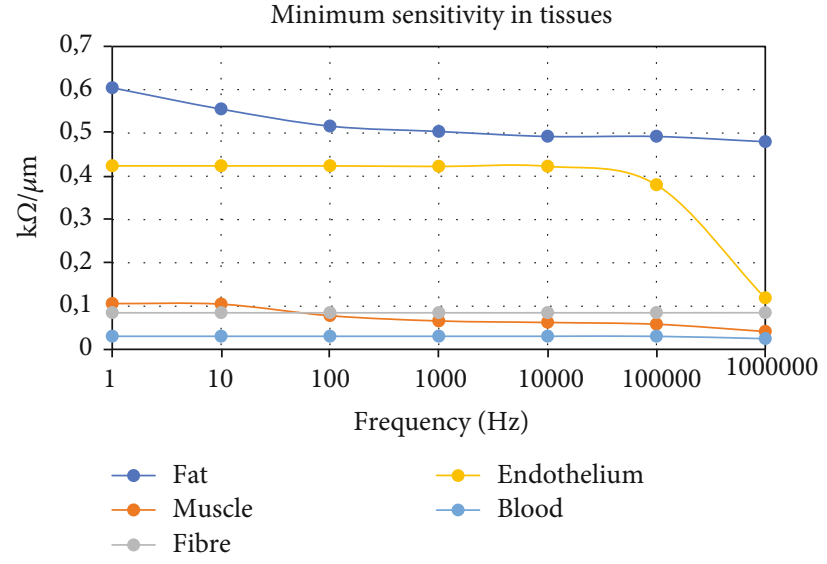

(b)

FIGURE 8: Maximum sensitivity of the impedance magnitudes for the tissues involved in restenosis using FEA: (a) maximum change ratio; (b) minimum change ratio.

TABle 3: Case definitions for FEA of restenosis inside stents considering several tissues involved in the neointimal layer and the resulting occlusion percentage calculated for a stent of $1150 \mu \mathrm{m}$ of the internal radius.

\begin{tabular}{lcccccc}
\hline & \multicolumn{5}{c}{ Thickness (in $\mu \mathrm{m})$} & Occlusion \\
Case & Fat & Muscle & Fibre & Endothelium & Blood & $(\%)$ \\
\hline 1 & 0 & 25 & 0 & 10 & 1115 & 3 \\
2 & 0 & 50 & 0 & 10 & 1090 & 5 \\
3 & 25 & 50 & 5 & 10 & 1060 & 8 \\
4 & 50 & 100 & 10 & 10 & 980 & 15 \\
5 & 100 & 100 & 20 & 10 & 920 & 20 \\
6 & 200 & 100 & 40 & 10 & 800 & 30 \\
7 & 250 & 175 & 75 & 10 & 640 & 44 \\
8 & 300 & 200 & 100 & 10 & 540 & 53 \\
9 & 400 & 300 & 100 & 10 & 340 & 70 \\
\hline
\end{tabular}

Table 3. Note that the expected maximum change in magnitude is in the range $(0.89,1.05) \mathrm{k} \Omega / \mu \mathrm{m}$ for all cases and frequency values, whereas the expected minimum change is between 28 and $33.3 \Omega / \mu \mathrm{m}$. The same information is provided in Figure 10(b) as a function of the lumen occluded percentage. These results are characterized by the following direct proportional relationships:

(i) We can expect a $1 \mu \mathrm{m}$ thickness increment per $1 \mathrm{k} \Omega$ increment

(ii) We can expect a $1 \%$ occlusion increment per $10 \mathrm{k} \Omega$ increment for the stent under study

3.3. Symmetrical Restenosis. Impedance changes were also analysed when both tissue thickness and frequency were modified on real stents. In this situation, the geometry is different from that of the previous sections. A stent is now placed around the tissues, which are concentric to it. Eight sets of four electrodes were considered. Each pair of electrodes (one for current excitation and the other for voltage measurement) was placed on an octagon vertex, as shown in Figure 11(b). These electrodes were separated by approximately the same distance as described in the above sections (see Figure 2). The tissue growth rate employed for FEA in this case follows the same patterns described in Table 3. For this reason, the impedance values obtained for the conditions described in this section are similar to those of the previous section. This is why we do not report them in this paper. Furthermore, the eight setups measured the same impedance because of the symmetrical geometry. All in all, Figure 9 shows a magnitude and phase similar to the values obtained for symmetrical restenosis.

3.4. Nonsymmetrical Restenosis. Given that symmetrical restenosis represents an ideal situation, we also tried to reproduce the impedance evolution when restenosis is not symmetrical with respect to the tissues, which is actually the case of real restenosis. The considered electrode setup is shown in Figure 12(a) along with the corresponding asymmetrical lumen, which was considered as a possible restenosis situation. The tissue profiles shown in Figures 12(b) and 12(c) correspond to significant increments of muscle and fat tissue, respectively. This means that in Figure 12(b), the muscle tissue has the highest influence on restenosis, while in Figure 12(c), restenosis is mainly caused by the fat layer. Five and six electrode sets were taken as a reference, corresponding to case 6 in Table 3 . This case was selected to prove that this technology can measure and identify whether the plaque is stable or vulnerable, even before a significant occlusion percentage is reached (50\% or more). Consequently, a smart stent could anticipate a real dangerous case, as fat plaques could be detached inside the blood vessels, leading to thrombosis. Any other real cases could also be described and solved using the same procedure.

Case 6 in Table 3 was simulated for the irregular lumens shown in Figures 12(b) and 12(c), in the same frequency range, considering an excess of muscle and fat, respectively. Figure 13 shows the magnitude and phase of the neointimal tissue BI obtained for an 8-electrode set at $1 \mathrm{kHz}$ and considering irregular and regular lumens. 


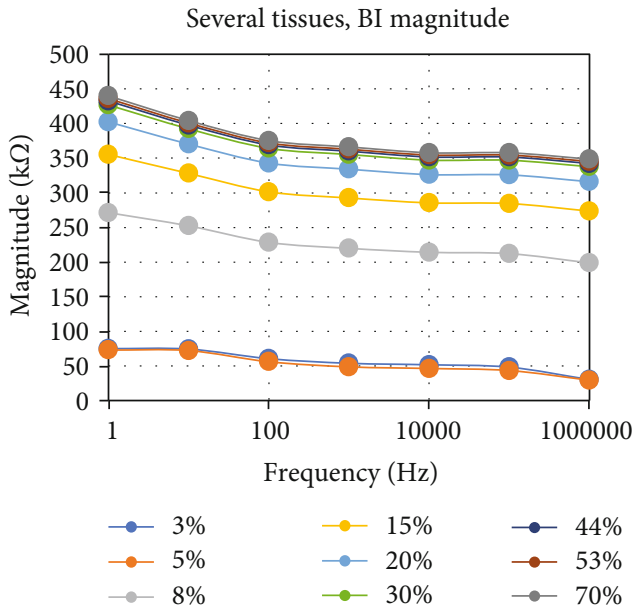

(a)

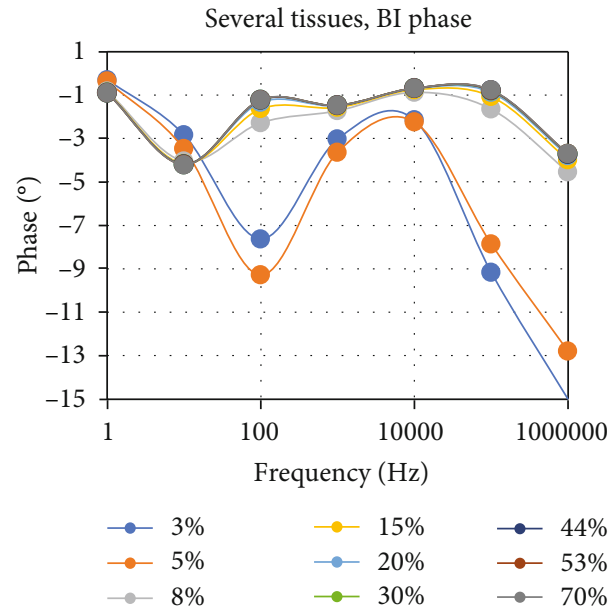

(b)

Figure 9: (a) BI magnitude versus frequency for several cases of tissues in Table 3 and (b) the corresponding phase impedance for the same cases.
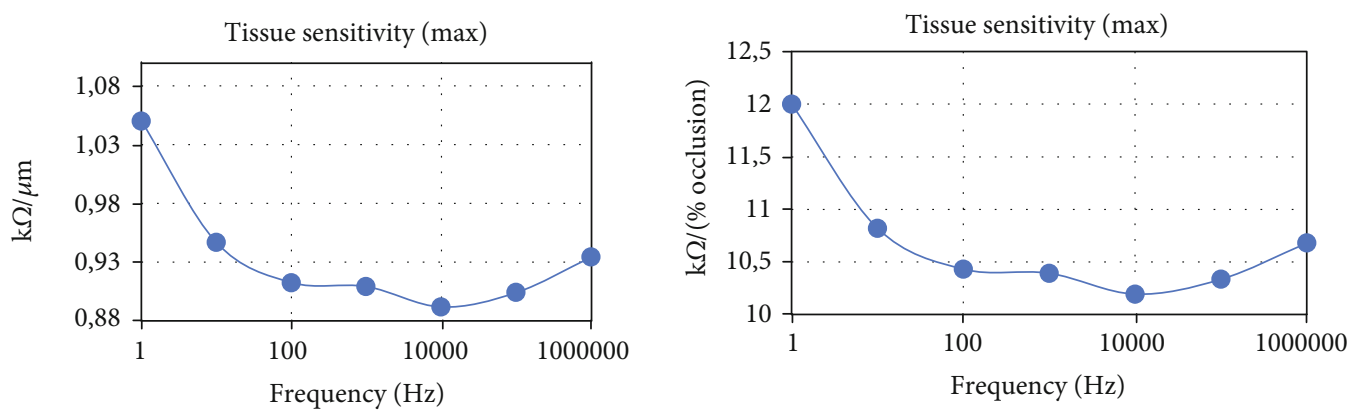

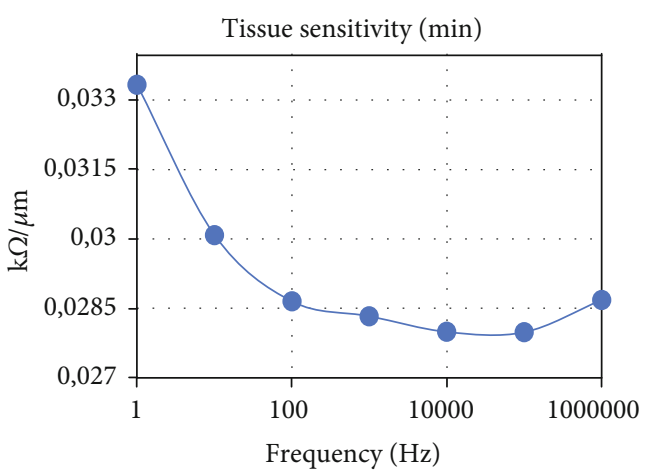

(a)

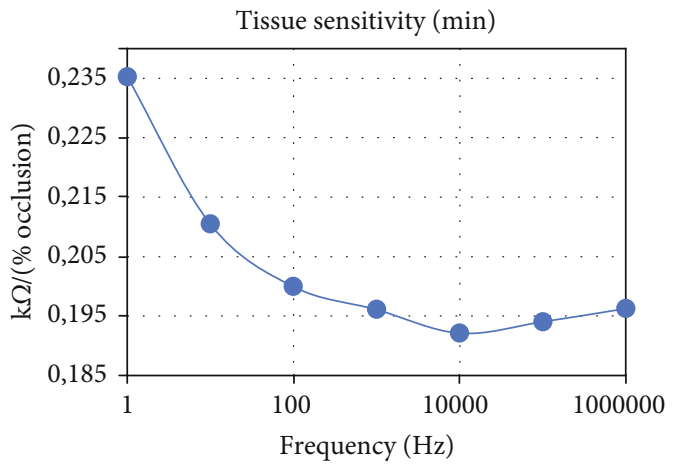

(b)

FIGURE 10: Maximum and minimum magnitude sensitivity of the BI observed for the cases listed in Table 3: (a) $\mathrm{k} \Omega / \mu \mathrm{m}$ and (b) $\mathrm{k} \Omega /(\%$ occlusion).

The results are similar for other frequencies. The regular or symmetrical lumen taken as a reference is represented in red. The stable (muscle layer takes precedence) and vulnerable [28] (fat layer prevails) plaque cases are represented in blue and black, respectively. Note that the impedance magnitude measured at the test points (1-8) decreases for lower dimensions of fat, while it increases for similar changes in muscle. In addition, the variation ranges for the absolute values of fat impedance magnitude are larger than those for the muscle. This impedance magnitude, which increases due to increments of fat tissue, represents a potential biomarker to detect vulnerable plaques in restenosis cases.

From the results shown in Figure 13, the increasing thickness of fat tissue can be predicted by observing the largest changes in BI magnitude, that is, from its maximum-tominimum values. However, smaller changes in BI magnitude could be caused by increments in thickness of muscle tissue 


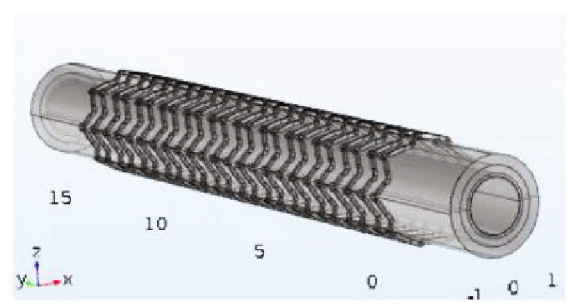

(a)

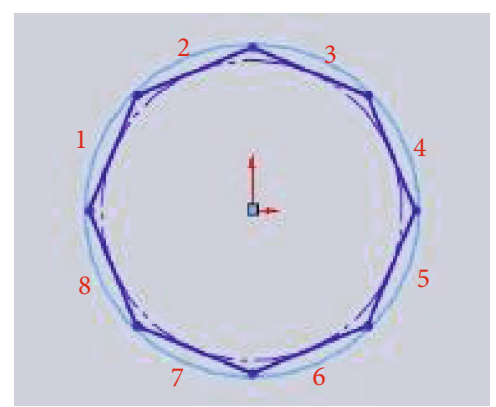

(b)

Figure 11: (a) Regular geometry stent, with an internal radius considered for the stent of $1150 \mu \mathrm{m}$, and (b) the 8 sets of two electrodes placed around and inside the stent contour.

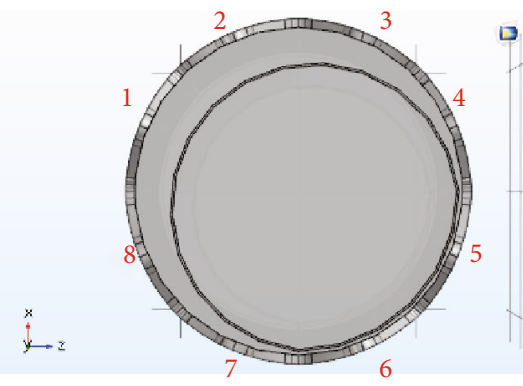

(a)

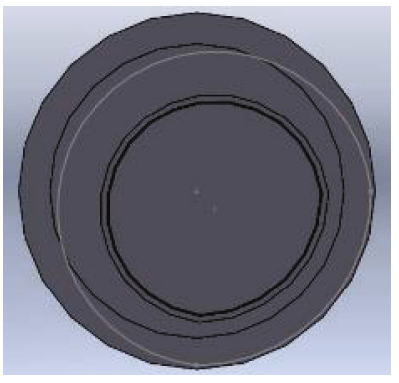

(b)

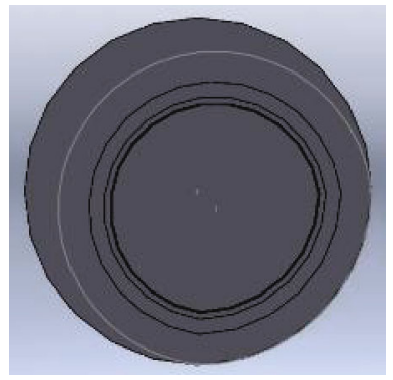

(c)

FIgURE 12: General irregular lumen inside a stent (a), generated by increments of muscle tissue (b) or increments of fat tissue (c).

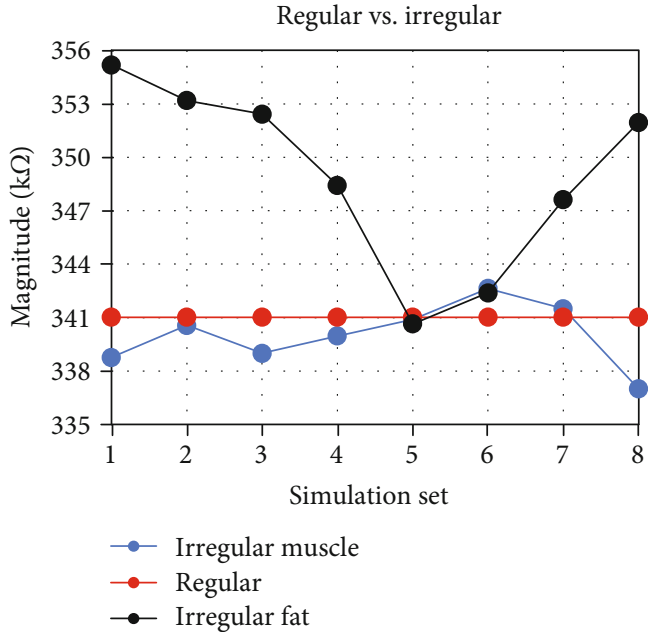

(a)

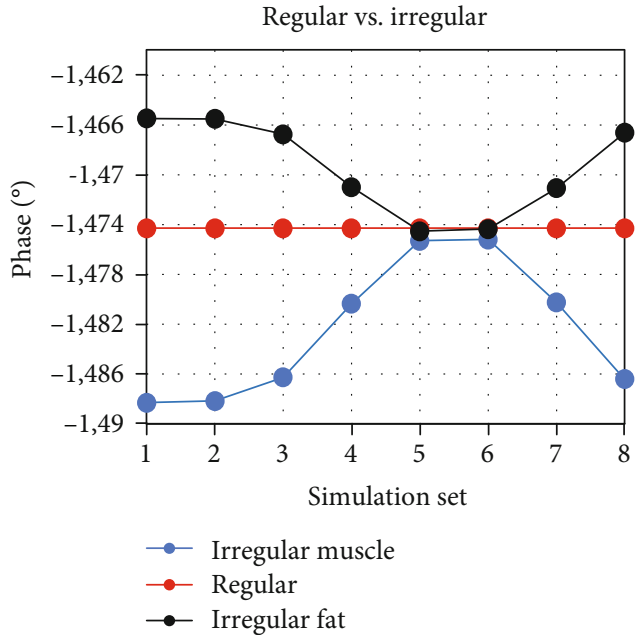

(b)

FIgURe 13: Magnitude measurements using FEA in regular and irregular lumens inside a stent at $1 \mathrm{kHz}$. The regular lumen (red) corresponds to case 6 in Table 3. The irregular lumen is caused by (blue) muscle increment (stable plaque) and (black) fat tissue increment (vulnerable plaque).

(below the reference values). For the analysed case, the occlusion of the stent is $30 \%$ and known a priori, but in a real situation, this percentage is actually the objective to be defined. In such cases, the BI magnitude increases more than $1 \%$ $(3.41 \mathrm{k} \Omega$ over $341 \mathrm{k} \Omega$, which is the approximated value of magnitude measured in set number 5), and it could be estimated as potential thickness increments of fat tissue and evaluated as a vulnerable plaque. This $1 \%$ value can be detected using reported approaches based on BI circuits [17].

\section{Conclusions}

This work presents a breakthrough in FEA applied to neointimal tissue characterization in implanted stents. It 
opens the door for real-time monitoring of lumens in implanted stents, thereby avoiding catheterism. A 4electrode setup has been proposed to minimize bioimpedance measurement leakage of the involved tissue layers and blood. The dependence of the different tissue layers involved in the neointimal composition is individually derived from the bioimpedance magnitude and phase for each tissue and for several representative restenosis situations. The results show that the increments of thickness in all tissues can be characterized through their corresponding changes in the magnitude of the impedance. The fat layer has the highest relevance on these impedance changes. For several FEA-based simulations of tissues, examples of both symmetrical and asymmetrical lumens have been considered. Our preliminary results demonstrate that it is possible to distinguish the growth of the different layers by detecting changes of $28 \Omega / \mu \mathrm{m}$ or $190 \Omega /(\%$ occlusion $)$ as the minimum values for the cases under study. In addition, for the analysed cases of multilayer and nonsymmetrical lumens, the proposed test allows distinguishing whether the main tissue involved in restenosis is fat or muscle, thereby enabling medical diagnosis assistance. Vulnerable or stable plaques in restenosis cases can be detected through the increment/decrement in magnitude impedance at $1 \mathrm{kHz}$ frequency.

\section{Data Availability}

The available data can be obtained from the corresponding author.

\section{Conflicts of Interest}

The authors declare that there is no conflict of interest regarding the publication of this paper.

\section{Acknowledgments}

This work was in part funded by the Spanish Government's Ministerio de Ciencia, Innovación y Universidades, Plan Estatal 2017-2020 Retos - Proyectos I+D+I: "Real Time Monitoring of Hemodynamic Variables Using Intelligent Stents (iSTENT) with Capacitive Sensors and Bioimpedance," under the project RTI2018-093512-B-C21, cofinanced with FEDER.

\section{References}

[1] OECD/EU, Health at a Glance: Europe 2016: State of Health in the EU Cycle, OECD Publishing, 2016.

[2] N. Townsend, L. Wilson, P. Bhatnagar, K. Wickramasinghe, M. Rayner, and M. Nichols, "Cardiovascular disease in Europe: epidemiological update 2016," European Heart Journal, vol. 37, no. 42, pp. 3232-3245, 2016.

[3] D. L. Kasper, A. S. Fauci, S. L. Hauser, D. L. Longo, and J. Larry, Eds., Harrison's Principles of Internal Medicine, McGraw Hill, New York, NY, USA, 19th Edition edition, 2016.

[4] T. J. Gundert, A. L. Marsden, W. Yang, D. S. Marks, and J. F. LaDisa, Jr, "Identification of hemodynamically optimal coronary stent designs based on vessel caliber," IEEE Transactions on Biomedical Engineering, vol. 59, no. 7, pp. 1992-2002, 2012.
[5] E. Y. Chow, A. L. Chlebowski, S. Chakraborty, W. J. Chappell, and P. P. Irazoqui, "Fully wireless implantable cardiovascular pressure monitor integrated with a medical stent," IEEE Transactions on Biomedical Engineering, vol. 57, no. 6, pp. 1487-1496, 2010.

[6] G. W. Stone, S. G. Ellis, L. Cannon et al., "Comparison of a polymer-based paclitaxel-eluting stent with a bare metal stent in patients with complex coronary artery disease: A randomized controlled trial," JAMA, vol. 294, no. 10, pp. 1215-1223, 2005.

[7] S.-J. Park, S. J. Kang, R. Virmani, M. Nakano, and Y. Ueda, "In-stent neoatherosclerosis: a final common pathway of late stent failure," Journal of the American College of Cardiology, vol. 59, no. 23, pp. 2051-2057, 2012.

[8] M. A. Clark, A. Bakhai, E. M. Pelletier, and D. J. Cohen, "Clinical and economic effects of coronary restenosis after percutaneous coronary intervention in a managed care population," Managed Care, vol. 14, no. 4, 2005.

[9] J. A. Miguel, D. Rivas, Y. Lechuga, M. A. Allende, and M. Martinez, "A novel computer-assisted design tool for implantable MEMS pressure sensors," Microprocessors and Microsystems, vol. 46, Part A, pp. 75-83, 2016.

[10] D. Rivas-Marchena, J. A. Miguel, Y. Lechuga, M. A. Allende, and M. Martinez, "Energy-efficient implantable transmitter for restenosis monitoring with intelligent-stents," in 2015 37th Annual International Conference of the IEEE Engineering in Medicine and Biology Society (EMBC), pp. 3323-3326, Milan, Italy, 2015.

[11] X. Chen, B. Assadsangabi, Y. Hsiang, and K. Takahata, "Enabling angioplasty-ready "smart" stents to detect in-stent restenosis and occlusion," Advanced Science, vol. 5, no. 5, article 1700560, 2018.

[12] T. Süselbeck, H. Thielecke, I. Weinschenk et al., "In vivo intravascular electric impedance spectroscopy using a new catheter with integrated microelectrodes," Basic Research in Cardiology, vol. 100, no. 1, pp. 28-34, 2005.

[13] F. Yang and R. P. Patterson, "A novel impedance-based tomography approach for stenotic plaque detection: A simulation study," International Journal of Cardiology, vol. 144, no. 2, pp. 279-283, 2010.

[14] L. Shedden, S. Kennedy, R. Wadsworth, and P. Connolly, "Towards a self-reporting coronary artery stent - measuring neointimal growth associated with in-stent restenosis using electrical impedance techniques," Biosensors and Bioelectronics, vol. 26, no. 2, pp. 661-666, 2010.

[15] P. K. Nair, J. G. Carr, B. Bigelow, D. L. Bhatt, Z. C. Berwick, and G. Adams, "LumenRECON Guidewire: Pilot study of a novel, nonimaging technology for accurate vessel sizing and delivery of therapy in femoropopliteal disease," Circulation: Cardiovascular Interventions, vol. 11, no. 1, article e005333, 2018.

[16] N. L. Opie, S. E. John, G. S. Rind et al., "Chronic impedance spectroscopy of an endovascular stent-electrode array," Journal of Neural Engineering, vol. 13, no. 4, 2016.

[17] G. Huertas, A. Maldonado, A. Yúfera, A. Rueda, and J. L. Huertas, "The bio-oscillator: a circuit for cell-culture assays," IEEE Transactions on Circuits and Systems-II-Express Briefs, vol. 62, no. 2, pp. 164-168, 2015.

[18] D. Rivas-Marchena, A. Olmo, J. Miguel, M. Martínez, G. Huertas, and A. Yúfera, "Real-time electrical bioimpedance characterization of neointimal tissue for stent applications," Sensors, vol. 17, no. 8, article 1737, 2017. 
[19] J. M. Portillo-Anaya, P. Pérez, G. Huertas et al., "Characterization of implanted stents through neointimal tissue bioimpedance simulations," in 41st IEEE Engineering in Medicine and Biology Society (EMBC), pp. 5625-5628, Berlin, Germany, 2019.

[20] I. Holland, C. McCormick, and P. Connolly, "Towards non-invasive characterisation of coronary stent reendothelialisation - an in-vitro, electrical impedance study," PLoS One, vol. 13, no. 11, article e0206758, 2018.

[21] H. P. Schwan and C. D. Ferris, "Four-electrode null techniques for impedance measurement with high resolution," Review of Scientific Instruments, vol. 39, no. 4, pp. 481-485, 1968.

[22] S. Grimnes and Ø. G. Martinsen, Bioimpedance and Bioelectricity Basics, Elsevier, 3rd edition, 2014.

[23] C. Gabriel and S. Gabriel, Compilation of the Dielectric Properties of Body Tissues at RF and Microwave Frequencies, 1996, http://niremf.ifac.cnr.it/docs/DIELECTRIC/Report.html.

[24] A. Olmo and A. Yúfera, "Computer simulation of microelectrode based bio-impedance measurements with Comsol," Biodevices, vol. 1, pp. 178-182, 2010.

[25] J. T. Dodge Jr., B. G. Brown, E. L. Bolson, and H. T. Dodge, "Lumen diameter of normal human coronary arteries. Influence of age, sex, anatomic variation, and left ventricular hypertrophy or dilation," Circulation, vol. 86, no. 1, pp. 232-246, 1992.

[26] D. A. Dowe, M. Fioranelli, and P. Pavone, Eds., Imaging Coronary Arteries, Springer, New York, NY, USA, 2nd edition, 2012.

[27] C. Stefanadis, C.-. K. Antoniou, D. Tsiachris, and P. Pietri, "Coronary atherosclerotic vulnerable plaque: current perspectives," Journal of the American Heart Association, vol. 6, no. 3, article e005543, 2017.

[28] A. L. Kierszenbaum and L. L. Tres, Histología y Biología Celular, Elsevier, España, 3a edition, 2012. 


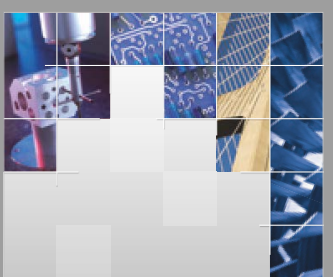

\section{Enfincering}
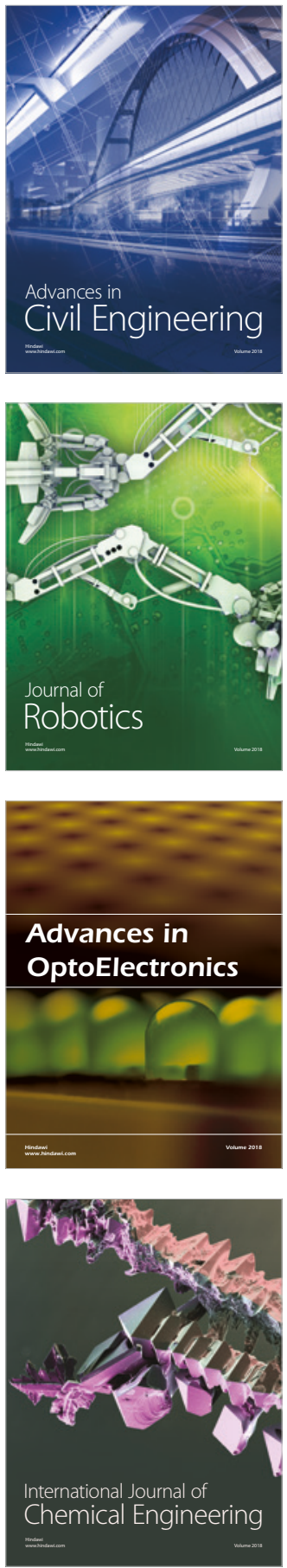

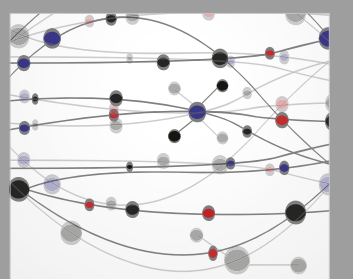

\section{Rotating \\ Machinery}

The Scientific World Journal

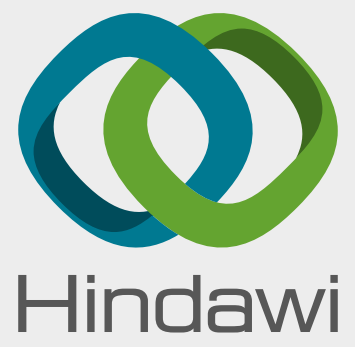

Submit your manuscripts at

www.hindawi.com
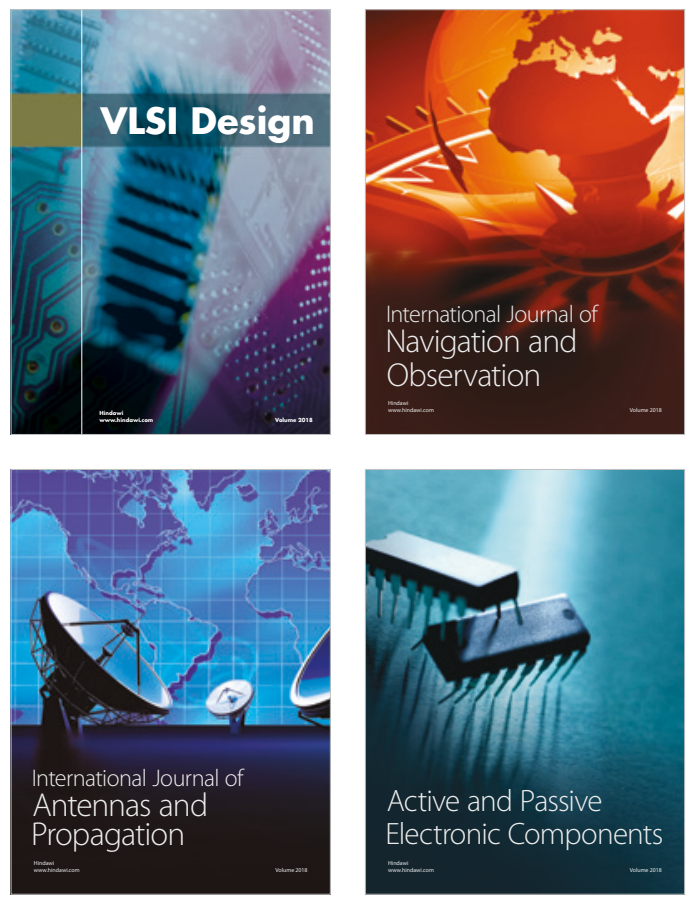
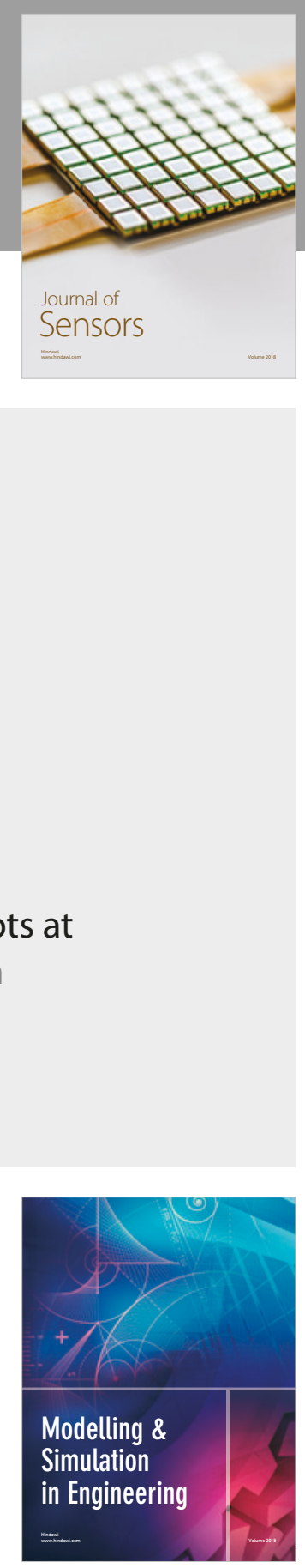

\section{Advances \\ Multimedia}
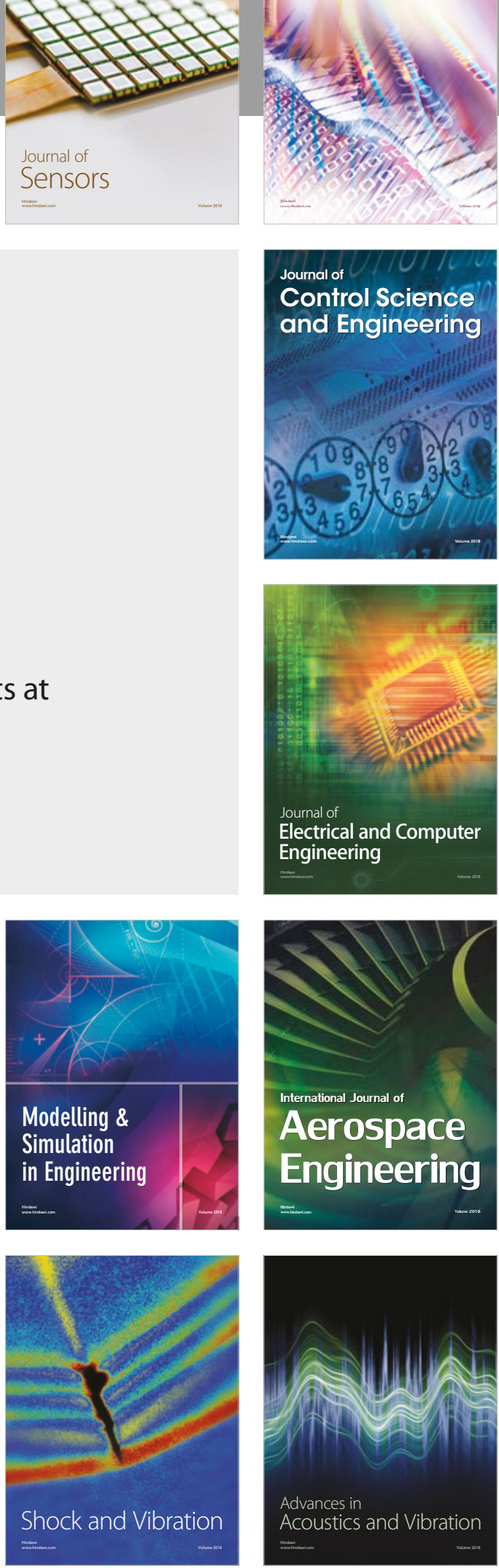